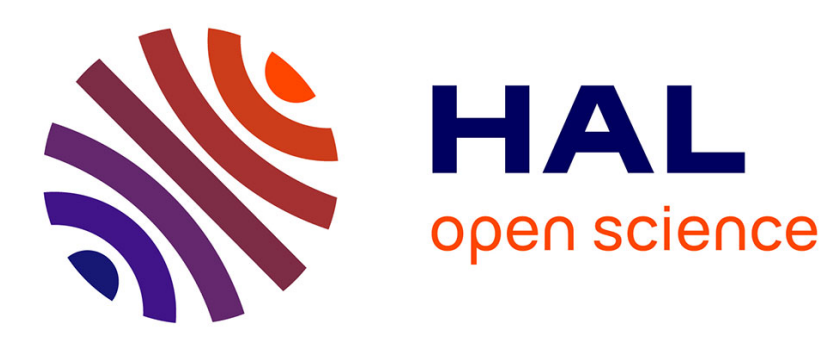

\title{
Evénement et conscience de l'événement dans le journal du concile Vatican II du sulpicien Marcel Breysse
}

\author{
Christian Sorrel
}

\section{To cite this version:}

Christian Sorrel. Evénement et conscience de l'événement dans le journal du concile Vatican II du sulpicien Marcel Breysse. Guillaume Cuchet, Charles Mériaux. La dramatique conciliaire de l'Antiquité à Vatican II, Presses universitaires du Septentrion, pp.441-456, 2019. halshs-02949785

\section{HAL Id: halshs-02949785 \\ https://shs.hal.science/halshs-02949785}

Submitted on 14 Mar 2021

HAL is a multi-disciplinary open access archive for the deposit and dissemination of scientific research documents, whether they are published or not. The documents may come from teaching and research institutions in France or abroad, or from public or private research centers.
L'archive ouverte pluridisciplinaire $\mathbf{H A L}$, est destinée au dépôt et à la diffusion de documents scientifiques de niveau recherche, publiés ou non, émanant des établissements d'enseignement et de recherche français ou étrangers, des laboratoires publics ou privés. 


\section{Guillaume Cuchet}

Charles Mériaux

(dir.)

\section{La dramatique conciliaire \\ de l'Antiquité à Vatican II}

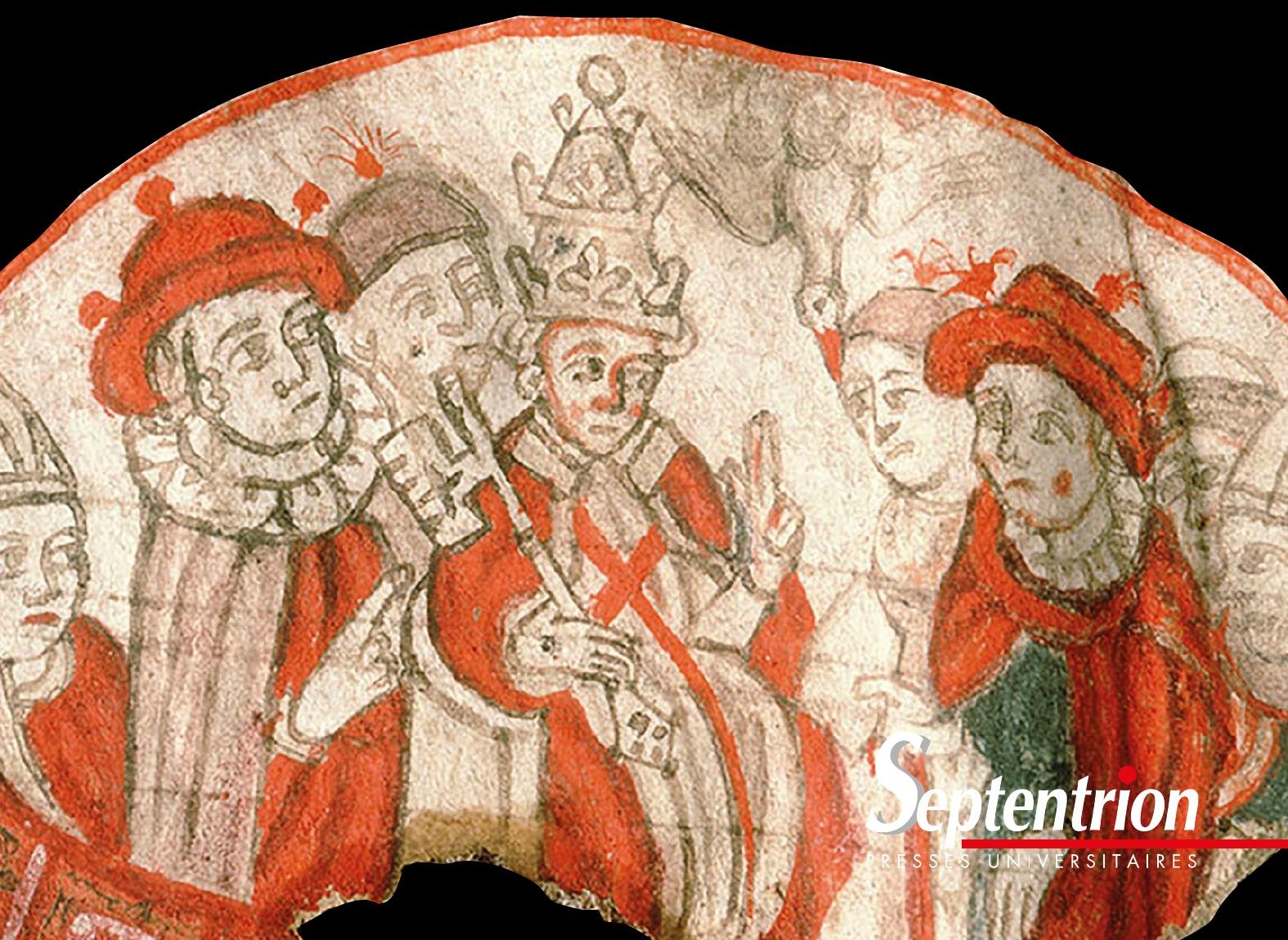




\section{La collection \\ Histoire et civilisations \\ est dirigée par \\ Carole Christen}

Cet ouvrage est publié après l'expertise éditoriale du comité

Temps, espace et société

et d'une double expertise externe.

Le comité est composé de :

- Frédéric Attal, Université Polytechnique des Hauts-de-France

- Xavier Boniface, Université de Picardie - Jules Verne

- Carole Christen (coordinatrice), Université de Lille

- Jean-François Condette, Université d'Artois et ESPE-Lille-Nord-de-France

- Thomas Golsenne, Université de Lille

- Jean Heuclin, Université Catholique de Lille

- Sandrine Huber, Université de Lille

- Yves Junot, Université Polytechnique des Hauts-de-France

- Hervé Leuwers, Université de Lille

- Judith Rainhorn, Université Paris 1 Panthéon-Sorbonne

-Éric Roulet, Université du Littoral - Côte d'Opale

- Emmanuelle Santinelli, Université Polytechnique des Hauts-de-France

- Arnaud Timbert, Université de Picardie - Jules Verne

- Béatrice Touchelay, Université de Lille

- Jérôme Vaillant, Université de Lille 


\section{Les Presses universitaires du Septentrion}

sont une association de cinq universités :

- Université de Lille,

- Université du Littoral - Côte d'Opale,

- Université Polytechnique Hauts-de-France,

- Université Catholique de Lille,

- Université Picardie Jules-Verne.

La politique éditoriale est conçue dans les comités éditoriaux.

Cinq comités et la collection « Les savoirs mieux de Septentrion » couvrent les grands champs disciplinaires suivants :

- Acquisition et Transmission des Savoirs,

- Arts et Littératures,

- Savoirs et Systèmes de Pensée,

- Sciences Sociales,

- Temps, Espace et Société.

Publié avec le soutien de la Communauté d'universités et d'établissements Lille Nord de France et de la Région Hauts-de-France.

(C) Presses universitaires du Septentrion, 2019 www.septentrion.com Villeneuve d'Ascq - France

\footnotetext{
Toute reproduction ou représentation, intégrale ou partielle, par quelque procédé que ce soit, de la présente publication, faite sans l'autorisation de l'éditeur est illicite (article L. 122-4 du Code de la propriété intellectuelle) et constitue une contrefaçon. L'autorisation d'effectuer des reproductions par reprographie doit être obtenue auprès du Centre Français d'Exploitation du Droit de Copie (CFC) 20 rue des Grands-Augustins à Paris.
} 


\section{Guillaume Cuchet, Charles Mériaux (dir.) avec les contributions de}

Silvia Acerbi, Antoine Arjakovsky, Philippe Blaudeau, Séverine Blenner-Michel,

Florence Close, Guillaume Cuchet, Davide Dainese, Thomas Deswarte,

Pierre Antoine Fabre, Loïc Figoureux, Étienne Fouilloux, Étienne Lock,

Catherine Masson, Sylvain Milbach, Hélène Millet, Nelson H. Minnich, Pascal Montaubin,

Martin Morard, Jacques Prévotat, François-Xavier Romanacce, Émilie Rosenblieh,

Philippe Roy-Lysencourt, Christian Sorrel, Ramón Teja, Sophie Vallery-Radot

\section{La dramatique conciliaire de l'Antiquité à Vatican II}

Publié avec le soutien de

I'Institut de Recherches Historiques du Septentrion (UMR 8529 - CNRS)

et de l'Institut universitaire de France 


\section{Politique de diffusion des livres numériques aux Presses universitaires du Septentrion}

Les Presses Universitaires du Septentrion mettent à la vente les livres numériques sur leur site internet sans appliquer de DRM (Digital Rights Management), ceci afin de ne pas réduire les usages de ses lecteurs. Leurs livres numériques n'en restent pas moins soumis au droit d'auteur.

En conséquence, les Presses universitaires du Septentrion demandent à leurs lecteurs de ne pas diffuser leurs livres numériques sur des plates-formes de partage ni de procéder à de multiples copies privées ( $>5$ ).

La violation des droits d'auteurs est constitutive du délit de contrefaçon puni d'une peine de 300000 euros d'amende et de 3 ans d'emprisonnement (CPI, art. L. 335-2 s.).

Le code de la propriété intellectuelle entend par contrefaçon tous les actes d'utilisation non autorisée de l'œuvre.

La loi incrimine au titre du délit de contrefaçon :

- " toute reproduction, représentation ou diffusion, par quelque moyen que ce soit, d'une œuvre de l'esprit en violation des droits de l'auteur, tels qu'ils sont définis et réglementés par la loi » (CPI, art. L. 335-3).

- "le débit [acte de diffusion, notamment par vente, de marchandises contrefaisantes], l'exportation et l'importation des ouvrages "contrefaisants" » (CPI, art. L. 335.2 al. 3). Source : http://www.culture.gouv.fr/Media/Thematiques/ Propriete-litteraire-et-artistique.

Pour plus d'informations, consultez le site internet des Presses Universitaires du Septentrion www.septentrion.com. 


\section{Table des matières}

Introduction

Guillaume Cuchet

\section{PremiÈRE PARTIE \\ ÉCRIRE ET RÉÉCRIRE LA DRAMATIQUE CONCILIAIRE}

Les conciles mis en textes,

ou comment le Saint-Esprit écrit droit avec des lignes courbes

Martin Morard

Nicée (325), ou l'invention de l'hérésie arienne 51

François-Xavier Romanacce

Éphèse I (431) et Éphèse II (449) : concilia ou latrocinia? .75

Ramón Teja, Silvia Acerbi

La réception spirituelle, mémorielle et historique du concile de Florence (1439).

Antoine Arjakovsky

Le problème de l'image dans le dernier acte du Concile de Trente : documents inédits du mois de novembre 1563

Pierre Antoine Fabre

\section{DEUXIÈME PARTIE}

LE PAPE ET LA PAPAUTÉ AU CCEUR DE LA DRAMATIQUE CONCILIAIRE

Sanctionner le pape sans rompre avec le Siège apostolique?

Retour sur la condamnation de Vigile prononcée

lors du concile de Constantinople II (553)

Philippe Blaudeau

Les conciles de la monarchie pontificale (1049-1312) :

des conciles sans drame?

Pascal Montaubin 
La condamnation de deux papes fauteurs

de schisme : un drame conciliaire (Pise, 1409)

\section{Hélène Millet}

La dramatique conciliaire à Constance (1414-1418) :

la fuite de Jean XXIII du concile.

\section{Sophie Vallery-Radot}

Le Saint-Esprit ou la légitimation de l'autorité conciliaire :

le procès du pape Eugène IV au concile de Bâle (1431-1439)

\section{Émilie Rosenblieh}

Dramatique inopportune : le concile du Vatican et l'opinion dans les pas de Mgr Dupanloup et de Louis Veuillot

Sylvain Milbach

\section{TROISIÈME PARTIE \\ L'EMPEREUR, LE ROI, L'ÉTAT, ACTEURS DE LA DRAMATIQUE CONCILIAIRE}

Le rôle de Constantin au concile de Nicée selon Eusèbe de Césarée. 259

\section{Davide Dainese}

Tolède III (589) et Tolède IV (633) :

deux conciles, deux conceptions du pouvoir

\section{Thomas Deswarte}

Le synode ecclésiastique de Francfort (794):

un concile carolingien bien inspiré ?

\section{Florence Close}

La résolution des conflits

entre la France et le Saint-Siège au Ve concile du Latran (1512-1517) 311

Nelson H. Minnich

Le Saint-Esprit corrigé ?

Le Saint-Siège face au concile provincial de Paris (1849-1850).

\section{Séverine Blenner-Michel}

$$
\begin{gathered}
\text { QuatRIÈme PARTIE } \\
\text { LA DRAMATIQUE PAR EXCELLENCE : } \\
\text { VATICAN II, LES MOMENTS, LES HOMMES, LES GROUPES }
\end{gathered}
$$

La « semaine noire » de Vatican II

\section{Étienne Fouilloux}

Henri de Lubac, un homme aux « ailes de géant » au concile Vatican II. 
L'intervention du cardinal Liénart au concile Vatican II le 13 octobre 1962....... 385

\section{Catherine Masson}

La Panafricaine épiscopale au Concile Vatican II :

simple tactique ou souci de représentativité ? 405

Étienne Lock

Les traditionalistes du Coetus Internationalis Patrum

dans la dramatique du concile Vatican II

Philippe Roy-Lysencourt

Événement et conscience de l'événement dans le journal du concile Vatican II du sulpicien Marcel Breysse 441

Christian Sorrel

Conclusion

Jacques Prévotat 


\title{
Événement et conscience de l'événement dans le journal du concile Vatican II du sulpicien Marcel Breysse
}

\author{
Christian Sorrel \\ Université Lyon 2 - LARHRA (UMR 5190)
}

L

'HISTOIRE DU CONCILE VATICAN II a déjà largement bénéficié de l'exploitation des journaux rédigés par les pères et les periti qui aident à restituer le déroulement des sessions, à préciser les intentions des acteurs et à analyser le cheminement des décisions, donc à appréhender l'événement, qui ne peut être réduit à sa production textuelle, aussi décisive soit-elle'. Mais beaucoup restent à découvrir, spécialement en France où leur inventaire est inachevé et où les éditions critiques sont rares, exception faite pour des figures majeures de la théologie ${ }^{2}$. C'est le cas des carnets du sulpicien Marcel Breysse, vicaire général de Mgr Couderc, évêque de Viviers, qui le choisit comme commensal et théologien personnel durant ses séjours romains et pour lequel il obtient le statut d'expert officiel peu après le début du concile³. Le prêtre ardéchois peut ainsi assister chaque matin aux congrégations générales dans la basilique Saint-Pierre et donner, depuis la tribune des periti, un témoignage précieux sur la dramatique conciliaire, tout en demeurant dans une position périphérique.

1 A. MelLon, «Les journaux privés dans l'histoire de Vatican II 》, dans M.-D. Chenu, Notes quotidiennes au Concile. Journal de Vatican II 1962-1963, Paris, 1995, p. 7-54.

2 Y. Congar, Mon journal du Concile, 2 t., Paris, 2002 ; H. DE Lubac, Carnets du Concile, 2 t., Paris, 2007 ; Carnets conciliaires de Mgr Auguste Jauffrès, ancien évêque de Tarentaise, Aubenas-surArdèche, 1992 ; " Journal conciliaire de Monseigneur Émile Blanchet. Première session 》, Transversalités, nº 121, 2012, p. 13-102 ; M. CANCOüet, L'Afrique au Concile. Journal d'un expert, Rennes, 2013.

3 Archives diocésaines de Viviers. Je remercie M. Daniel Gay, archiviste diocésain, qui a attiré mon attention sur ce document et rendu possible sa consultation. 


\section{- Le témoignage de M. Breysse}

Toute la carrière de Marcel Breysse (1903-1978) a pour cadre le diocèse de Viviers dont il est originaire et pour lequel il est ordonné prêtre en septembre 1926, à la veille de son entrée dans la Compagnie de SaintSulpice qui l'envoie à Rome, après son année de Solitude, pour préparer un doctorat en théologie à l'Angelicum (1927-1929). De 1929 à 1958, il enseigne au petit puis au grand séminaire d'Aubenas et en 1958, Mgr Couderc (1882-1968), évêque depuis 1937, lui confie la charge de vicaire général, qu'il garde jusqu'en 1977. En octobre 1962, il lui demande de l'accompagner à Rome : " Je ne me fis pas prier [...]. Sans doute se rendait-il compte que sa théologie - étudiée autrefois à Rome - lui était devenue lointaine et approximative », commente l'intéressé4. Très vite, Mgr Couderc s'emploie à le faire nommer peritus en s'adressant au cardinal Cicognani, secrétaire d'État, par l'entremise de Mgr Gouet, directeur du secrétariat de l'épiscopat français. II obtient satisfaction et, le 10 novembre, $M$. Breysse rejoint pour la première fois la tribune réservée aux experts, même s'il ne prête serment qu'en septembre $1963^{5}$.

Peritus, il ne l'est en fait qu'en titre, bien conscient d'être peu préparé "à la grande leçon de théologie que devait être le concile " ${ }^{6}$. En quatre ans, il participe à une seule réunion d'experts au Séminaire français sur le schéma XIII et s'éclipse " discrètement » : « Les personnalités présentes m'en ont imposé, et je me suis senti mal à l'aise : Le Guillou, Lubac, Frisque, Martelet, Denis, Subil, Hua. ${ }^{7} "$ "Jamais personne ne m'a rien demandé ", ajoute-t-il lors de la dernière session au cours de laquelle il se sent toujours « tout petit à contempler les aigles qui tournoyaient si haut au-dessus de [sa] tête » pendant une conférence du père Chenu ${ }^{8}$. II s'efforce en revanche de jouer son rôle d'expert privé auprès de Mgr Couderc. Mais sa tâche se révèle malaisée face à un prélat habitué à commander et à garder le secret, malgré le naufrage progressif de ses capacités intellectuelles qui conduit le pape Paul VI, alerté par l'épiscopat français et le nonce Bertoli, à exiger sa démission au cours de l'été 1965 , tout en acceptant de la différer jusqu'au terme de la dernière session.

4 Journal, «Pages préliminaires » (1966).

5 Ibid., 29 octobre et 10 novembre $1962 ; 28$ septembre 1963.

6 Ibid., « Pages préliminaires » (1966).

7 Ibid., 21 septembre 1964.

8 Ibid., 22 septembre et 12 octobre 1965.

9 Mgr Couderc, opéré d'urgence d'une hernie à Rome, n'assiste en fait qu'au début de la session et rentre dès la fin octobre 1965 à Viviers, où il remet sa démission le 14 décembre 
M. Breysse essaie d'éclairer son évêque sur les enjeux majeurs du concile en préparant des notes, par exemple sur le rapport entre Écriture et Tradition aux sources de la Révélation, lieu d'un des premiers affrontements doctrinaux en 1962. Mais Mgr Couderc se contente en général d'une «simple lecture » des schémas, tel le De Beata Maria Virgine : «Tout cela est ponctué à la fin de chaque phrase par "bien entendu, évidemment, à la bonne heure". Pas une seule fois, il n'a été besoin d'expliquer, ni même d'amorcer une discussion $»^{10}$. Il entend cependant multiplier les vota sur des questions secondaires et sollicite son théologien pour les mettre en forme. Celui-ci voudrait le dissuader, avant de céder. « J'obéis passivement, et je revêts de somptuosité latine cette pauvreté ", écrit-il à propos d'un règlement de vie pour les professeurs de petit séminaire "d'une banalité comparable à celle des lettres pastorales " ${ }^{11}$. Parfois, en proie à une " colère contagieuse ", il tape le texte "tel quel, avec ses maladresses, ses incorrections, ses mots impropres », tout en corrigeant "quelques barbarismes " ${ }^{12}$. Mais en novembre 1963, il tente d'éviter une prise de parole dans l'aula en alertant plusieurs évêques, avant d'être rassuré par Mgr Vignancour, évêque de Valence, convaincu de l'imminence de la clôture du débat ${ }^{13}$.

Le cas Couderc est sans doute singulier, sans être unique. «Schémas et chapitres dansent dans sa tête une sarabande endiablée ", affirme M. Breysse en s'interrogeant dès 1962 sur sa maîtrise de la procédure de vote par placet, non placet et placet juxta modum ${ }^{14}$. "C'est un homme seul, qui porte sa détresse avec lui et qu'une dignité et une fierté mal placées empêchent de se confier et de se faire aider », ajoute-t-il deux ans plus tard avant d'obtenir la confidence de la nullité systématique des votes émis au début du concile en cochant une case et en barrant les deux autres ${ }^{15}$. De cette expérience, partagée avec des confrères comme l'abbé Denis, secrétaire du cardinal Gerlier, découle un scepticisme relatif sur la mécanique conciliaire : "Espérons, sans en être bien sûr, que nos évêques votent en pleine connaissance de cause ", écrit-il en 1964, alors que le rythme de travail s'accélère ${ }^{16}$. II s'inquiète des effets

et est remplacé le jour même par Mgr Hermil, auxiliaire d'Autun, pressenti durant l'été.

M. Breysse reste à Rome jusqu'à la clôture du concile.

10 Journal, 14 octobre 1962.

11 Ibid., 5 octobre 1963.

12 lbid., 5 novembre 1962.

13 Ibid., 27 novembre 1963.

14 Ibid., $7^{\text {er }}$ et 30 novembre 1962.

15 Ibid., 25 septembre 1964 et 11 octobre 1965.

16 Ibid., 29 septembre 1964. 
d'entraînement dont Mgr Couderc est l'acteur et la victime. " Je ne reconnais plus notre évêque : Rome est l'ennemi contre lequel il faut s'insurger. Rome, c'est la curie, mais aussi le pape tant qu'il sera italien", constate-t-il à la fin de la première session ${ }^{17}$. Et il reprend deux ans plus tard à propos du contrôle des naissances : "Dès qu'un collègue a parlé, alors les thèses les plus hardies ne l'effraient plus. La peur d'être seul de son avis ? $»^{18}$ Mais cette pente, qu'il lie aussi à l'ignorance, n'est pas irréversible et la même année, Mgr Couderc se réjouit du rejet du mariage des diacres après l'ordination, coup de frein au « débordement de nouveautés » : " Le vieil évêque ultra-traditionnel, un tantinet rétrograde, comment a-t-il pu faire jusqu'ici pour marcher avec ses collègues français ? C'est un bel exemple de sociologie contraignante », commente M. Breysse ${ }^{19}$.

Le sulpicien, engagé dans un tête-à-tête pesant avec son évêque, auquel il offre même sa démission en 1964, trouve une compensation dans le séjour romain, rythmé par les promenades quotidiennes dans la cité et les excursions de fin de semaine dans les environs après le travail en aula. Il est en effet assidu aux congrégations générales. Observateur fin, bon latiniste, il avoue toutefois ne pas toujours saisir « ce qui se dit » et mesure les limites de sa position, même s'il est un privilégié par rapport aux autres prêtres de Viviers : "Les appréciations des confrères sur les événements et les hommes du concile me laissent souvent rêveur et au fond m'amusent. On porte sur le pavois et on met plus bas que terre tels ou tels, selon qu'ils plaisent ou non, selon les jugements rapides d'une presse avide de sensationnel [...]. Qui sait si je ne m'abuse pas aussi ? II y a tant de choses que j'ignore ", confie-t-il après la première session ${ }^{20}$.

Il est vrai que M. Breysse est isolé dans la Rome conciliaire. Avec Mgr Couderc, il réside à la maison du Cénacle, sur le Janicule, qui accueille peu de pères, sinon le nonce au Pérou, Mgr Carboni, qui " parle beaucoup pour ne dire que ce qu'il veut ", et l'archevêque de Pittsburgh, Mgr Wright, membre important de la Commission doctrinale, "caustique " pour ses collègues ${ }^{21}$. Ses contacts avec les évêques français sont ponctuels, autant que ses échanges avec des

\footnotetext{
Ibid., 8 décembre 1962.

8 Ibid., 7 novembre 1964.

19 Ibid., 29 novembre 1964.

20 Ibid., 31 janvier 1963.

21 Ibid., 14 novembre 1962 et 14 septembre 1964.
} 
personnalités comme le père Wenger ${ }^{22}$ ou Mgr Martimort, pessimiste sur l'issue du concile en raison de la personnalité de Paul VI, « sensible aux influences ", «qui ne sait pas décider $»^{23}$. II rencontre un peu plus souvent ses confrères sulpiciens, M. Michalon, « source jaillissante de nouvelles », et surtout le supérieur de la Compagnie Girard et le procureur Géraud, dont il est loin cependant de partager la « hargne » contre les pères et la " pagaille introduite dans le gouvernement de l'Église par la prolongation du concile $»^{24}$.

Le journal de M. Breysse donne en effet l'image d'un prêtre ouvert et prudent, favorable aux évolutions en cours, ironique pour la « théologie à la manière du musée des âmes du purgatoire » chère à son évêque, prompt à déplorer " la place excessive que tient l'Écriture sainte dans les schémas $»^{25}$. Fils d'une terre de confrontation et de cohabitation confessionnelles, il est ouvert à l'œcuménisme dont il fut un précurseur dans son diocèse. Le 11 octobre 1962, lors de la cérémonie inaugurale, il regrette que le pape prête serment "séparément des autres évêques": "Je pense aux frères séparés que la formule choquera. ${ }^{26} \|$ est en relation avec le pasteur Roux, admis au concile comme observateur, mais stigmatise " la hargne protestante » et le " comportement huguenot » auxquels ce dernier, déçu par le tour des débats, cède parfois ${ }^{27}$. II salue l'attitude des frères de Taizé, tout en s'interrogeant sur leur logique en observant Max Thurian devant la chapelle du Saint-Sacrement à SaintPierre : « II est resté en prière silencieuse pendant quelques instants debout en dehors de la barrière de séparation [...]. Aurait-il fait un pas de plus vers la doctrine eucharistique? Aurait-il fait ce geste ce matin s'il n'avait pas été seul ? $\$^{28} \mathrm{Ce}$ souci d'orthodoxie, joint à une réelle modération, est tout aussi évident à propos des définitions mariales, de la morale familiale ou de la théologie du sacerdoce qui lui donne l'occasion de défendre l'École française contre «l'activisme et le vide spirituel »

22 Absent de Rome pendant quelques jours en novembre 1964, le père Wenger demande à M. Breysse d'aider Noël Copin à préparer les comptes rendus des congrégations générales pour La Croix.

23 Journal, 11 novembre et 7 octobre 1964.

24 Ibid., 24 octobre 1964 et 12 octobre 1965. On trouve le même constat sous la plume du dominicain Labourdette à propos de M. Girard : «Encore un qui est catastrophé, presque scandalisé, de l'attitude des évêques, des réactions à l'égard de la curie romaine, où il voit de la hargne » (journal, 2 novembre 1962).

25 Ibid., 20 septembre 1964.

26 Ibid., 11 octobre 1962.

27 Ibid., 24 novembre 1962.

28 Ibid., 4 octobre 1963. 
envahissants ${ }^{29}$. Mais s'il s'inquiète des tensions et des dérives, il n'en reste pas moins fidèle à la posture conciliaire qu'il a adoptée dès les premiers jours et que son admission dans l'aula a confirmée.

\section{- Regards sur l'aula depuis la tribune des periti}

En accédant à la tribune des periti à la veille du premier affrontement doctrinal sur le De fontibus Revelationis, M. Breysse prend sans délai la mesure de l'assemblée, marquée par une alternance de grisailles et de fulgurances. Un « morne ennui » caractérise les premiers jours, malgré un effort d'attention soutenu, alors que le débat sur le De sacra liturgia se termine : «Les haut-parleurs et les micros sont parfaits, les orateurs ne le sont pas. » La séance du 14 novembre consacrée au nouveau schéma est en revanche « splendide » : " Le concile s'est réveillé [...]. L'atmosphère était lourde quand le cardinal Ottaviani s'est levé pour présenter le rapport préalable [...]. J'étais personnellement atterré [...]. À mesure que parlaient les cardinaux Liénart, Frings, Alfrink, Ritter, Léger, Bea, je sentais croître mon exaltation », confie M. Breysse ${ }^{30}$.

Tout le journal est rythmé par des propos similaires qui donnent le pouls de l'aula. Si les « séances sensationnelles » ne manquent pas, les interventions s'épuisent en " répétitions fastidieuses » et les matinées se succèdent, " ennuyeuse ", " morne », « longue et pénible », « longue et

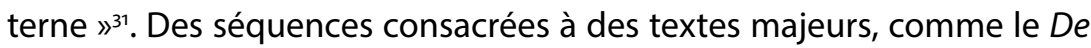
Revelatione, se révèlent au final riches, malgré des baisses de tension : "Ce fut en somme un beau débat. Les positions nettes et franches, les compréhensions différentes ont apporté beaucoup d'intérêt aux discussions. $1^{32}$ Mais d'autres textes sont moins mobilisateurs, tel le De vita et ministerio sacerdotali auquel le sulpicien est attentif : « La pauvreté de la discussion sur le sacerdoce a été affligeante [...]. Les questions matérielles d'honoraires à établir ou de servantes à payer sont abordées de la manière la plus basse et la plus vulgaire [...]. Comment veut-on que le peuple chrétien et les mécréants eux-mêmes éprouvent de la vénération pour le sacerdoce quand les évêques n'en donnent pas l'exemple ? \$33 $^{33}$

D'une session à l'autre, le climat évolue, stimulant à l'automne 1964, quand des mesures sont prises pour accélérer le déroulement du concile, lassant à l'automne 1965, quand les pères se contentent d'écouter les

29 Ibid., 15 octobre 1965.

30 lbid., 13 et 14 novembre 1962.

31 Ibid., 4 et 10 octobre $1963 ; 22$ septembre et 29 octobre 1964 .

32 Ibid., 6 octobre 1964.

33 Ibid., 13 et 14 octobre 1964 
rapports et de voter, "occupation austère $»^{34}$. Chaque session a aussi ses temps où l'on « sent la fatigue », où l'on « se déplace » et « cause par petits groupes » et où l'on semble prêt « à signer n'importe quoi pour avoir [la] liberté $»^{35}$. L'observateur extérieur qu'est M. Breysse est toutefois conscient qu'il est « difficile de juger des dispositions de l'assemblée d'après ce qu'on entend en congrégation générale ». II cherche donc à deviner les stratégies qui s'y déploient, par exemple lors du débat sur la collégialité : «Du côté des partisans [...], les intentions ne sont pas toujours d'une pureté théologique parfaite. La collégialité risque de devenir un bélier par lequel on veut enfoncer le mur des congrégations romaines, réduire tout au moins leur puissance et leurs attributions. On comprend aussi peut-être que, de l'autre côté, la primauté du Saint-Père n'est pas défendue pour elle-même, mais quelquefois pour l'avantage des défenseurs. ${ }^{36}$

Quoi qu'il en soit, le caractère répétitif de nombre d'interventions laisse du temps à l'expert pour promener son regard sur l'aula et saisir des moments qui caractérisent la scène conciliaire. La liturgie matinale, célébrée selon divers rites, est l'occasion de réflexions souvent critiques, au moins au début, même s'il reconnaît de la piété « à ce vieux prélat tremblotant qui revendique âprement sa place, la première, et récite longuement son bréviaire pendant la messe, élévation incluse, et pendant la discussion conciliaire » : «Que fait-il ? prie-t-il ? écoute-t-il ? La piété romaine peut concilier bien des choses apparemment incompatibles. $\|^{37} \mathrm{~A}$ la veille de la clôture de la première session, " c'est le brouhaha et le branle-bas des soldes dans un grand magasin ", tandis que les évêques " pratiquent la chasse aux images ${ }^{38}$. Avec le temps, les auditrices, d'abord " fort édifiantes », apparaissent « intrigantes et bavardes », faisant " avec des sourires la nique aux règlements et à la délicatesse » pour accueillir leurs relations dans leur tribune et pérorer ${ }^{39}$. M. Breysse ne manque pas d'épingler dans le même temps les manies des prélats, tels le préfet de la Congrégation des séminaires et universités Pizzardo et son secrétaire Staffa : "Quand il approche midi, [Mgr Staffa] se rend à la Confession, derrière la table de présidence, s'accoude nonchalamment à la balustrade et attend. Quelques minutes après, de son pas de

\footnotetext{
34 Ibid., 15 septembre 1964 et 29 octobre 1965.

35 Ibid., 12 novembre 1964 et 5 octobre 1965.

36 Ibid., 16 octobre 1963.

37 Ibid., 10 novembre 1962.

38 Ibid., 7 décembre 1962.

39 Ibid., 2 octobre 1964 ; 4 et 6 décembre 1965.
} 
nonagénaire, le cardinal Pizzardo le rejoint, et tous deux quittent l'aula, serviette sous le bras. ${ }^{40}$ Mais l'attention que le sulpicien porte aux détails se fait aussi quête de signes et de sens lorsqu'il note les conciliabules entre les pères, à l'exemple des cardinaux Siri et Ruffini, «surtout quand un évêque italien n'est pas de la note classique », les absences, les silences ou les inflexions des discours : «Pourquoi [Felici] a-t-il été absent une bonne partie de la séance ? » ; " Pourquoi Carli se tait-il ? Pourquoi Florit a-t-il changé d'avis ? »" "Scruter les hésitations ou les distractions de quelques Éminences, voire de quelques Béatitudes, présente un certain intérêt », explique-t-il devant la volte-face du cardinal tanzanien Rugambwa lors d'un vote par assis et debout en $1964^{42}$.

Le sulpicien accorde, on le voit, une grande importance aux personnalités qui animent l'aula et goûte l'affrontement des ténors. Comme bien d'autres, il porte un regard acéré sur les curialistes et les chefs de la minorité, en premier lieu le cardinal Ottaviani, secrétaire du Saint-Office et président de la Commission doctrinale ${ }^{43}$. "Le schéma et le cardinal seraient-ils une même chose, tant il me semble faire corps avec son œuvre? Est-il personnellement mis en cause ? Serait-il le Christ poursuivi par la haine de ses frères ? ", se demande $M$. Breysse après avoir écouté le rapport introductif du De Ecclesia en 1962. "Ce qui me déplaît surtout chez [lui], c'était, encore plus marqué que l'an dernier, c'est la persuasion, transparente à travers ses phrases, qu'il incarne l'orthodoxie, que tout ce que pensent les autres doit se référer, comme une norme, à ce que pense le secrétaire du Saint-Office ", reprend-il un an plus tard, lorsque ce dernier conteste la validité du vote d'orientation du 30 octobre 1963 sur le texte révisé du De Ecclesia ${ }^{44}$. Le jugement est un peu moins sévère pour le cardinal Ruffini, archevêque de Palerme, et le cardinal Siri, archevêque de Gênes. Le premier, orateur "à la manière claire et précise ", " dit d'excellentes choses, mais bien traditionnelles » sur les laïcs pour apparaître au final « mesquinement rétrograde » sur le mariage ${ }^{45}$. Le second, "d'une force logique étonnante », avance des « affirmations

\footnotetext{
40 Ibid., 8 novembre 1963.

41 Ibid., 6 novembre 1963 et 2 octobre 1964. Mgr Felici est le secrétaire général du concile, Mgr Carli, évêque de Segni, est l'un des porte-parole de la minorité et Mgr Florit est archevêque de Florence.

42 Ibid., 9 novembre 1964.

43 C. Sorret, "Le concile d'un évêque missionnaire. Mgr Michel Bernard, archevêque de Brazzaville, à Vatican II », dans L'Afrique et la mission. Terrains anciens, questions nouvelles avec Claude Prudhomme, éd. O. SAaidia et L. ZerBinI, Paris, 2015, p. 115-124.

44 Journal, $1^{\text {er }}$ décembre 1962 et 8 novembre 1963.

45 Ibid., 16 octobre $1963 ; 25$ septembre et 29 octobre 1964 .
} 
plus impressionnantes que les critiques incisives du cardinal Ruffini », sans éviter tout propos " désagréable et injuste » lors du débat sur le diaconat permanent ${ }^{46}$. Mgr Carli, évêque de Segni et compagnon de route du supérieur des Spiritains Lefebvre au sein du Coetus internationalis Patrum, ne mérite en revanche aucune indulgence avec « sa voix aigre et dénuée de sympathie » : « Est-il possible d'être intelligent et bête à la fois à ce degré ? », écrit M. Breysse après son intervention sur l'objection de conscience en $1965^{47}$.

Le sulpicien est souvent plus bref pour présenter les ténors de la majorité. II « admire [la] fermeté de pensée et [le] courage » du cardinal Frings, archevêque de Cologne, et apprécie le verbe « clair et incisif » du cardinal Léger, archevêque de Montréal, en qui «l'humilité et la vérité vont bien ensemble $»^{48}$. II estime aussi le cardinal Lercaro, archevêque de Bologne, «théologien profond et précis », qui apparaît cependant " difficile à comprendre » au fil des sessions, parce qu'il est « tout en nuances » et " n'indique pas où il va $»^{49}$. II est plus réservé à l'égard du cardinal Suenens, archevêque de Malines, «I'homme des grandes constructions idéales », " percutant et écouté », mais discutéso. II salue enfin les cadets de la majorité, tel Mgr De Smedt, évêque de Bruges, dont l'« appel à la conscience des pères » contre le De fontibus Revelationis le conduit au bord des larmes, mais qui le déçoit par le ton « acerbe et passionné » de sa deuxième intervention, une vraie " diatribe " $^{51}$. " Il y a des gens qui, une fois qu'ils se sont fait un nom, peuvent se payer le luxe de se faire applaudir hors de propos ", commente M. Breysse, qui remarque aussi I'année suivante le nouvel auxiliaire de Bologne, Mgr Bettazzi, animé par " une fougue de jeunesse fort sympathique au demeurant $»^{52}$.

À l'aune de ces personnalités, la scène conciliaire française apparaît décevante et le jugement du sulpicien se fait sévère, en phase avec les pères Congar et de Lubac ${ }^{53}$. II déplore en premier lieu le " bavardage

46 Ibid., 15 octobre 1963 et 15 septembre 1965.

47 Ibid., 16 septembre et 7 octobre 1965.

48 Ibid., 14 octobre et 25 novembre $1963 ; 21$ septembre $1964 ; 15$ septembre 1965.

49 Ibid., 12 novembre 1963 et 28 septembre 1964.

50 Ibid., 4 décembre 1962 et 12 novembre 1963. Les Français reprochent notamment au cardinal Suenens sa conception extensive de l'Action catholique, identifiée à l'apostolat des laïcs.

51 Ibid., 19 novembre et $1^{\text {er }}$ décembre 1962. Nombre de témoins jugent sévèrement la seconde intervention de Mgr De Smedt, à l'instar de Mgr Bernard, archevêque de Brazzaville (journal, $7^{\text {er }}$ décembre 1962).

52 lbid., 11 octobre 1963.

53 F. Le Moigne et C. Sorrel, "Les évêques français et le concile Vatican II », Anuario de historia de la Iglesia, t. 21, 2012, p. 185-205; C. SorReL, « Un acteur important du concile Vatican II : 
facile des évêques » : "Six évêques français ont parlé ce matin ; nous n'aurions certainement rien perdu à n'en entendre que trois », affirme-til en $1964^{54}$. Il est vrai que rares sont ceux dont le « niveau » semble « à la hauteur » des enjeux, tels le cardinal Lefebvre, «à [la] manière grande et sereine », Mgr Ancel, auxiliaire de Lyon, qui « donne plus de prix à ce qu'il dit » par son " accent de sincérité », « rare au concile », Mgr Guerry, archevêque de Cambrai, et Mgr Garrone, archevêque de Toulouse : « lls sont phraseurs en général, et on ne sent guère de souffle apostolique dans leurs grandes théories pastorales. Vraiment et sincèrement, je ne suis pas fier de notre épiscopat. $\$^{55}||$ reproche aux uns leur ignorance du latin (Stourm, Sauvage), aux autres leur « voix claironnante » mise au service " d'insignifiantes banalités » (Urtasun) et le cardinal Liénart lui-même, " aimablement banal ", n'est pas épargné, sans parler des vieillards réduits à une " piètre exhibition » ou provoquant « de la pitié » (Rastouil, Gerlier ${ }^{56}$ ). II stigmatise plus encore le déficit de compétence théologique de beaucoup et dénonce, avec à peine plus de modération que MM. Girard et Géraud, leur propension à suivre les théories nouvelles : "Le Monde est leur bréviaire et leur directeur de conscience. C'est du moins ce que l'on dit. Je crois plutôt que c'est de leur part sotte vanité de ne pas paraître en retard. ${ }^{{ }^{57}}$ De ce point de vue, Mgr Marty, archevêque de Reims, dont il critique la conception du sacerdoce, symbolise à ses yeux les qualités et les défauts de l'épiscopat français : "Quel homme charmant, simple, tout proche des humbles et des petits. Mais quel ignorant ! Et quel danger que l'on ait chargé cet homme d'un rapport sur le sacerdoce $»$, conclut-i|is8.

Si le sulpicien privilégie logiquement ses compatriotes, il s'intéresse aussi aux représentants d'autres épiscopats. II n'apprécie guère les Orientaux, qui " ressemblent à une meute déchaînée » contre la latinité et s'enlisent « dans les problèmes de personnes, d'influences et de

Mgr Garrone, archevêque de Toulouse », dans B. BARBiche, C. SorRel (dir.), La France et le concile Vatican II, Paris/Bruxelles, 2013, p. 139-172.

54 Journal, 5 novembre 1963 et 22 septembre 1964.

55 Ibid., 28 novembre 1962, 7 octobre 1963 et $1^{\text {er }}$ octobre 1965.

56 Ibid., 23 novembre 1962 ; 7 et 17 octobre $1963 ; 28$ septembre et 14 novembre 1964 ; 30 septembre 1965.

57 Ibid., 12 octobre 1965.

58 Ibid., 17 octobre 1965. Mgr Marty est le rapporteur du schéma De ministerio et vita presbyterorum. II défend « la théorie du sacerdoce-mission » qui semble « bien courte, peu ontologique » à M. Breysse, partisan de fonder «le sacerdoce sur le caractère du sacrement, comportant configuration au Christ souverain prêtre ». 
préséances $»^{59}$. II s'emporte contre les Américains, par exemple le cardinal Cushing, archevêque de Boston, «parlant si fort que tout se brouillait », ou Mgr Sheen, auxiliaire de New York, qui provoque « une violente envie de rire »: «De l'orateur de télévision, il avait tous les trucs et toutes les faiblesses; on frémissait à ses intonations pathétiques, on entendait ses émotions, ses respirations, ses soupirs et ses silences. ${ }^{60} \|$ évoque des figures singulières, tel l'évêque d'Aquino Musto, au " prophétisme intempérant » contre l'œcuménisme, ou le prélat bénédictin Ammann, " hurluberlu » emporté par une "violente diatribe contre les nonces $»^{6}$. II restitue ainsi par touches la physionomie des congrégations générales et propose des clés pour appréhender les sommets dramatiques dont il entrevoit la portée pour le destin du concile sans pouvoir dénouer tous les fils de sa position d'observateur quelque peu marginal.

\section{Scénarios de crise}

M. Breysse fréquente l'aula depuis moins de dix jours lorsqu'il est témoin de la première crise décisive qui se joue, du 19 au 21 novembre 1962, autour du scrutin d'orientation sur le De fontibus Revelationis, dont beaucoup de pères souhaitent la réécriture contre l'avis du parti curial, attaché aux schémas élaborés durant la phase préparatoire. L'ambiguïté de la question posée et la confusion qui en découle ne permettent pas toutefois de dégager la majorité des deux tiers requise pour un rejet du texte et le débat doit se poursuivre, malgré le malaise de l'assemblée. Mais l'intervention de Jean XXIII, qui renvoie le texte devant une commission mixte issue de la Commission doctrinale et du Secrétariat pour l'unité des chrétiens, désamorce la crise et offre aux pères des potentialités inédites ${ }^{62}$.

Sous le regard du sulpicien, qui découvre les rouages de l'assemblée, l'épisode apparaît comme un théâtre d'ombres et s'identifie surtout à l'agitation de l'aula. Celle-ci est visible dès la fin de la séance du 19 : «Pendant que parlait Mgr Ancel, à la table de présidence, on pouvait voir des discussions animées. Le secrétaire général [Felici] allait de l'un à l'autre, d'un groupe à l'autre... Que se passait-il ? » Le 20, un peu mieux informé par les conversations de l'après-midi et de la soirée, il s'attend à une séance " orageuse » : «Par indiscrétion, on savait que

59 Ibid., 16 et 19 octobre 1964.

60 Ibid., 23 septembre et 9 novembre 1964. Mgr Sheen est effectivement un spécialiste des médias.

61 Ibid., 3 décembre 1962 et 16 octobre 1963.

62 G. Alberigo et É. Foullooux (éd.), Histoire du concile Vatican II, t. II, Paris, 1998, p. 281-320. 
l'on voterait. » Une fois encore, les mouvements l'alertent dès avant le scrutin : "On avait vu le cardinal Ruffini s'agiter, parler avec animation avec ses voisins, se déplacer pour entretenir l'un ou l'autre de ses collègues de la présidence. » L'annonce de la question ("La discussion doit-elle être interrompue ? ») est loin de calmer le jeu et, en quelques minutes, tout bascule ${ }^{63}$ :

Quand le secrétaire [Felici] eut lu la question une seconde fois, [Ruffini] demanda la parole pour expliquer qu'en somme « interrompu » voulait dire « retarder » sine die, c'est-à-dire rejeter de telle sorte qu'il ne soit plus jamais question de ce schéma. Personne du reste ne rectifia son interprétation. Mais aussitôt, sensation, agitation, conversations houleuses, discussions. Ottaviani descend de sa place, aborde les uns et les autres [...]. II vient à la table de présidence, dit quelques mots au cardinal Ruffini, puis disparaît [...]. Les évêques, pendant ce temps, ont commencé à voter et, dans la confusion, protestent qu'ils n'ont pas compris le sens et la portée de la question. Le secrétaire les apaise en leur disant qu'ils auront de nouveaux bulletins de vote, mais ils ne devront pas déchirer les premiers. Pourquoi ? C'est le brouhaha des assemblées populaires; un bon quart d'heure se passe dans cette confusion anarchique. Enfin, le président de tour, le cardinal Frings, rappelle tout le monde à l'ordre et déclare qu'en attendant les résultats du scrutin, il convient de passer à la suite, c'est-à-dire à l'examen du schéma, bien que, dit-il, son opinion personnelle eût été d'interrompre.

Le sulpicien tente aussitôt de décrypter la scène en mettant en avant les rumeurs sur les «frictions » au sein du conseil de présidence dont il croit identifier un indice dans le flottement autour des résultats du vote, prévus d'abord pour le lendemain, puis annoncés en fin de séance. II s'interroge sur l'emploi du " mot "interrompre" pour "rejeter" » : « Est-ce un scrupule canonique ? une formule diplomatique qui semble mieux respecter les droits du Saint-Père et se tient ainsi hors de portée des canons ecclésiastiques ? »Quoi qu'il en soit, les problèmes

63 La formulation initiale ("La discussion sur le schéma doit-elle continuer ou non ? ») a été abandonnée après l'intervention du cardinal Ruffini auprès du conseil de présidence qui aurait accepté ce changement pour éviter, en cas de rejet, « une offense trop directe au cardinal Ottaviani » selon le cardinal Liénart, interrogé par le recteur de l'Institut catholique de Paris. La confusion s'installe dès lors : «Si le schéma ne nous plaît pas, il faut répondre placet et, s'il nous plait, il faut répondre non placet », écrit Mgr Blanchet ( " Journal conciliaire... », loc. cit., p. 79). Au final, 1368 des 2209 pères présents demandent l'interruption du débat, tandis que 822 veulent sa poursuite : la majorité des deux tiers (1473) requise pour le rejet du texte n'est pas atteinte. 
lui apparaissent entiers, celui « de l'atteinte aux prérogatives pontificales ${ }^{64}$, celui d'un "schéma dont une minorité impose la discussion à une majorité qui n'en veut pas ». II accueille donc favorablement, le 21, la décision de renvoi prise par Jean XXIII, sans savoir s'il y a eu un recours auprès de lui ou s'il a agi de sa propre initiative. Il se réjouit autant de la réserve de l'aula en ces circonstances - ni applaudissement, ni « réaction apparente », hormis l'afflux de visiteurs au bar - et relit le résultat de la veille comme un signe : "Peut-être valait-il mieux que les adversaires du schéma n'aient pas atteint la majorité des deux tiers. Dans le cas contraire, on aurait pu attaquer le vote comme attentatoire aux droits du Saint-Père. Après avoir tant exalté ces mêmes droits, ils ne peuvent que s'incliner sans aucune réticence, du moins ne pourraient-ils pas l'exprimer sans se contredire. $\nu^{65}$

Cette attitude ne traduit aucune réserve de $M$. Breysse face au projet réformateur, mais exprime une préoccupation d'unité dont témoigne sa réaction à l'affrontement qui avait opposé quatre jours plus tôt le cardinal Doepfner, archevêque de Munich, au cardinal Ottaviani : "Comment sortira-t-on de cet imbroglio ? Aux yeux du monde, c'est, si l'on veut, un signe de liberté de pensée et d'expression ; mais il faudrait aussi ne pas oublier le signe de l'unité. $\nu^{66}$ La seconde session, qui voit la cristallisation de la majorité, tend pourtant à le rassurer. Le 30 octobre 1963, il se réjouit du résultat du vote d'orientation, impatiemment attendu, sur le De Ecclesia ${ }^{67}$, et son exaltation est à la mesure de la tension des jours précédents :

L'atmosphère était pessimiste ce matin [...]. En regardant vers la table de présidence, je guettais des signes qui auraient pu être une confirmation, et tout le monde autour de moi faisait comme moi [...]. Enfin, on annonce le résultat : stupeur !... Plus de $80 \%$ de suffrages favorables! Ce n'est pas possible [...]. Rome et l'Italie ont vécu sur leur vieille théologie de 1900, aux arêtes vives et établies pour l'éternité. Rome et l'Italie ne se sont pas aperçues qu'une autre théologie de l'Église naissait à l'étranger et était en train de conquérir le monde sur les points précis de l'épiscopat et du laïcat. Rome aurait pu payer très cher son ignorance systématique de ce qui se dit ailleurs, et l'Église catholique elle-même, par contrecoup, s'il n'y avait pas le concile. Heureuse Église catholique, à qui le Seigneur a donné Jean XXIII [...].

64 Les partisans du schéma jugent impossible le rejet d'un texte approuvé par le pape.

65 Journal, 19-21 novembre 1962.

66 Ibid., 17 décembre 1962.

67 G. Alberigo et É. Foullouux (éd.), Histoire du concile Vatican II, t. 3, op. cit., Paris, 2000, p. 76-119. 
Le concile est somme toute un merveilleux agent d'unité [...]. Je suis maintenant optimiste.

Le sulpicien se demande toutefois "Mais que va faire la vieille garde ? $»^{68}$ et reste attentif aux signes donnés par l'aula, alors qu'il saisit mieux son fonctionnement et est mieux informé. Il apparaît dès lors plus nuancé dans ses jugements, comme le montrent ses réactions lors de la « semaine noire » du concile (14-21 novembre 1964), épreuve pour la majorité69. Le 14, il se réjouit de la distribution des modi du De Ecclesia, saluée par des « applaudissements vigoureux »: "Elle signifiait non seulement que le texte était débloqué, mais que l'on s'en tenait au texte de la commission. Les craintes de ces derniers jours se dissipaient. » Le 16, la communication de la Nota explicativa praevia sur le rapport entre collégialité épiscopale et primauté pontificale ne le trouble pas : «[Elle] n'apporte pas de lumière nouvelle, redit ce que les partisans de la collégialité ont exprimé si souvent, que la collégialité n'existe et ne peut exister qu'avec le pape [...]. Le texte me paraît intact et plus éclairé. $»^{70}$ L'information, donnée par des évêques français et par Mgr Wright, selon laquelle le texte a été approuvé par la Commission doctrinale le rassure encore. Mais le 17 au matin, le père Labourdette l'alerte sur le mécontentement de nombre de pères que confirme l'atmosphère « houleuse » de l'aula: «On allait, on venait, on se cherchait, des groupes discutaient avec animation [...]. II y avait manifestement de l'électricité dans l'air. » Le faible nombre d'opposants au chapitre 3 l'apaise cependant : «Chose curieuse, que je relate comme je l'ai éprouvée, ces applaudissements vigoureux me paraissaient sereins et exprimer davantage la joie d'une unanimité retrouvée que la victoire d'un parti ou d'une idée. ${\aleph^{71}}^{11}$

$M$. Breysse attend dès lors dans le calme le vote du De libertate religiosa, dont il s'étonne qu'il ne puisse pas faire l'objet de modi, compte tenu de l'ampleur des changements depuis la version précédente. II est donc favorable à un report à la session suivante et regrette, le 18, que le conseil de présidence et les modérateurs ne le décident pas d'emblée pour s'en remettre au choix des pères eux-mêmes : « Je redoute [...] une aile marchante américaine, attachée sentimentalement à un succès immédiat [...]. Ils voteront par entraînement, sans savoir ce qu'ils ont voté ni pourquoi. » Le 19, il se trouve en revanche en phase avec la

68 Journal, 30 octobre 1963.

69 G. Alberigo et É. Foullzoux (éd.), Histoire du concile Vatican II, t. 4, op. cit., Paris, 2003, p. 473-553.

70 Journal, 14-16 novembre 1962.

71 Ibid., 17 novembre 1962. 
majorité de l'assemblée, dépossédée de toute initiative après l'annonce de l'annulation du vote par le cardinal Tisserant, doyen du Sacré Collège et président du conseil de présidence, et il décrit la tempête qui soulève la basilique Saint-Pierre ${ }^{72}$ :

Stupeur, puis agitation indescriptible. Beaucoup d'évêques quittèrent leur place pour tenir des conciliabules, et le ton de voix n'était pas toujours modéré. Je regardais attentivement le conseil de présidence. À la nouvelle [qu'il] avait pris cette décision, le cardinal Meyer [archevêque de Chicago] s'étonna. Je le vis questionner Ruffini [...]. À trois reprises, Meyer quitta sa place pour aller s'expliquer avec le cardinal doyen. Meyer était blême et Tisserant levait les bras de temps en temps comme pour dire : qu'y puis-je maintenant ? [...]. La conversation était générale, des groupes se formaient, se défaisaient pour se reformer avec d'autres [...]. Puis Mgr De Smedt lut le rapport qu'on lui concédait de lire. On l'écouta avec tension, profitant de toutes les occasions pour manifester [...]. Quand il eut terminé, les applaudissements durèrent longtemps ; s'éteignant ici, ils reprenaient plus loin, comme des vagues successives. Le modérateur du jour, le cardinal Doepfner, dut attendre longtemps avant que le flot fût passé [...]. Je partageais personnellement cette émotion, non sur le fait, mais sur le mode [...]. La mesure qui vient d'être prise, quel qu'en soit l'auteur, est très regrettable, Tisserant, Cicognani ou le pape lui-même. Il fallait s'en tenir à ce qu'on avait dit la veille. Sinon, on semble déposséder le concile d'un droit qu'on lui avait d'abord déféré. C'est malheureux et maladroit.

L'émotion est encore amplifiée par l'annonce de modifications, "venant de l'autorité supérieure », au schéma De oecumenismo, soumis au vote le lendemain : «En réalité, les modifications, sauf une ou deux, étaient de minime importance ; mais dans l'incapacité de vérifier, on se laissait aller aux suppositions les plus alarmistes. $1^{73}$ Le 20, M. Breysse, sévère pour le cardinal Tisserant et la rivalité entre les organes dirigeants du concile, écoute encore "les bruits les plus incontrôlables [...] sur la genèse de l'incident », mais il juge que « l'orage s'apaise »74. Et son appréciation est balancée, lorsque Paul VI, lors de la cérémonie de clôture du 21, proclame Marie Mater Ecclesiae, une démarche mal reçue

72 É. Foulloux, Eugène, cardinal Tisserant 1884-1972. Une biographie, Paris, 2011, p. 640-641. Sans avoir pu avertir tous les membres du conseil de présidence, le cardinal Tisserant exécute la décision du tribunal administratif du concile saisi par des pères qui mettent en avant les transformations du texte pour réclamer un nouvel examen, mais dont beaucoup sont hostiles à la liberté religieuse, thème sensible et controversé.

73 Journal, 19 novembre 1964.

74 Ibid., 20 novembre 1964. 
par une fraction de la majorité comme par les observateurs, à l'instar du pasteur Roux qui lui confie « sa tristesse et son découragement ». Tout en estimant que l'œcuménisme consiste " d'abord à se montrer tel que l'on est », il juge que ce titre "n'ajoute rien au dogme catholique, ni à la manière de faire ou de vivre des catholiques ", d'autant que la "réalité est contenue depuis longtemps dans d'autres titres incontestables»: «Fallait-il faire tant de bruit pour proclamer Marie Mère de l'Église ? [...]. Était-il convenable d'y insister au point d'en faire l'objet de la moitié du discours du pape ? Sans doute, du point de vue doctrinal, il avait raison contre certains pères ou théologiens timorés qui soulevaient des objections spécieuses. [Mais] le triomphe aurait dû être modeste ", conclut-il en observant la ferveur démonstrative du peuple romain ${ }^{75}$.

Lire la dramatique conciliaire à travers le journal de Marcel Breysse, c'est bien sûr l'observer selon un angle particulier que l'auteur, réformateur pondéré, témoin privilégié plus que peritus, reconnaît après la cérémonie de clôture du 8 décembre 1965 : "Ainsi finit mon journal du concile. C'est moi, autant et peut-être plus que le concile, que j'y ai inséré. ${ }^{76}$ Mais ce regard spécifique, aussi fragmenté et partiel, sinon partial, qu'il soit, n'en est pas moins précieux pour comprendre l'histoire de Vatican II, qui ne peut être réduite aux seize textes promulgués, comme le sulpicien croit utile de l'écrire au terme de la "semaine noire » de 1964 : « Les incidents [...] passeront et seront oubliés, tandis que les décrets conciliaires resteront [...]. Seulement là où sont les décisions conciliaires, là est le Saint-Esprit. Les agitations connexes sont des réactions humaines étrangères aux vrais problèmes. » $^{77}$ Acte de foi rassurant de quelqu'un qui s'interroge à d'autres moments sur la présence de l'Esprit dans l'assemblée ${ }^{78}$ ? Sans doute. II n'empêche que tout le journal fait figure de démenti à cette affirmation : I'histoire des conciles est l'histoire des hommes et des circonstances qui les ont portés autant que l'histoire des textes qu'ils ont élaborés.

\footnotetext{
Ibid., 21 novembre 1964.

Ibid., 11 décembre 1965.

Ibid., 22 novembre 1964.

78 « Je sais que l'Esprit saint se sert de tout, même de nos ignorances, mais j'ai de la peine à le trouver et à l'adorer dans le calme quand je me trouve dans cette situation », ibid., 23 octobre 1963.
} 



\section{La dramatique conciliaire de l'Antiquité à Vatican II}

Guillaume Cuchet, Charles Mériaux (dir.)

\section{Disponible en versions PDF et ePub}

sur le site des Presses universitaires du Septentrion

PDF - ISBN 978-2-7574-2816-0 : $26 €$

ePub - ISBN 978-2-7574-2825-2: $26 €$

Lot papier + numérique - ISBN 978-2-7574-2834-4 : $38 €$

\section{Retrouvez-nous}

sur www.septentrion.com

sur notre page Facebook

et sur Twitter

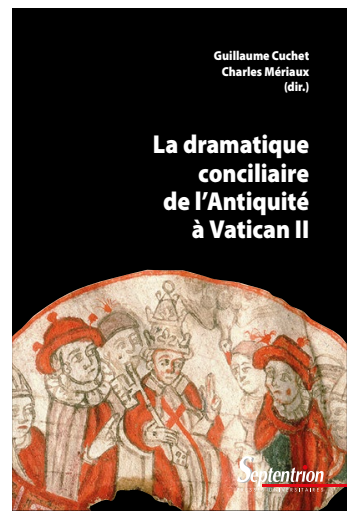




\section{Ouvrage composé par}

Émilie Duvinage

\section{Ouvrage réalisé avec}

La chaîne d'édition XML-TEI Métopes

Méthodes et outils pour l'édition structurée

avec la/les police(s) : Myriad Pro

\section{Dépôt légal}

septembre 2019

$1895^{\mathrm{e}}$ volume édité par

les Presses universitaires du Septentrion

59654 Villeneuve d'Ascq - France

\section{Septentrion}


Guillaume Cuchet est professeur d'histoire contemporaine à l'université Paris-Est Créteil. II travaille sur l'histoire et l'anthropologie religieuse des sociétés contemporaines.

Charles Mériaux est professeur d'histoire du Moyen Âge à l'université de Lille. II est spécialiste de I'histoire religieuse du haut Moyen Âge occidental.

Contributeurs Silvia Acerbi • Antoine Arjakovsky • Philippe Blaudeau • Séverine Blenner-Michel • Florence Close - Guillaume Cuchet • Davide Dainese • Thomas Deswarte • Pierre Antoine Fabre • Loïc Figoureux • Étienne Fouilloux - Étienne Lock • Catherine Masson • Sylvain Milbach • Hélène Millet • Nelson H. Minnich • Pascal Montaubin - Martin Morard • Jacques Prévotat • François-Xavier Romanacce • Émilie Rosenblieh • Philippe Roy-Lysencourt - Christian Sorrel • Ramón Teja • Sophie Vallery-Radot.

\section{La dramatique conciliaire de I'Antiquité à Vatican II}

En bonne théologie chrétienne, les conciles sont censés être «inspirés 》 ou «assistés » par le Saint-Esprit, mais force est de constater que leurs annales sont pleines de conflits, coups de théâtre, manœuvres, aussi bien dans la conduite des assemblées que la rédaction des documents qui en sont issus. Vues de près, les opérations du Saint-Esprit paraissent bien humaines. Cet ouvrage étudie, dans la longue durée et à des échelles différentes, les ressorts historiques de cette «dramatique conciliaire ». Comment comprendre que les acteurs des conciles aient pu être à la fois des tacticiens rompus aux usages de la mécanique des assemblées et des croyants sincères, convaincus que le Saint-Esprit pilotait en sous-main les opérations et que ce combat en apparence politique était en réalité spirituel ? Qu'est-ce que ces convictions nous disent de la nature de ces assemblées si particulières que sont les conciles?

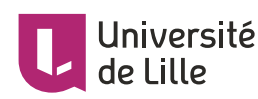
de Lille

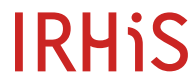

Institut de Recherches
Historiques du Septentrion

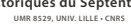

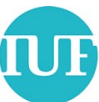

institut universitaire de France
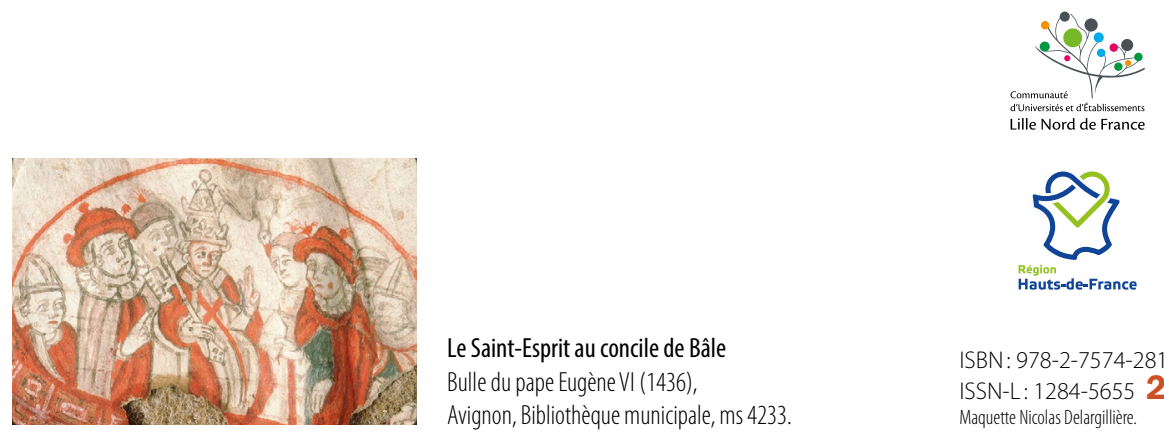

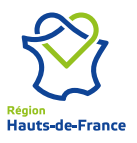

